Mathematical Research Letters 2, 161-169 (1995)

\title{
INTEGRATION BY PARTS FOR PINNED BROWNIAN MOTION
}

\author{
O. EnCHEV AND D. STROOCK
}

\begin{abstract}
A в Stract. In [D2], Driver showed how to adapt the techniques which he introduced in [D1] in order to develop an integration by parts formula on the space of pinned Brownian paths on a manifold. Here, it is shown how to carry out the analogous program using the ideas introduced in [ES]. The result derived here is slightly sharper than Driver's and, perhaps, a little more readily applicable.
\end{abstract}

\section{Pinned Brownian motion}

Let $M$ be a compact, connected, $d$-dimensional Riemannian manifold; denote by $\mathcal{O}(M)$, with fiber map $\pi: \mathcal{O}(M) \longrightarrow M$, the associated bundle of orthonormal frames $\mathfrak{e}: \mathbb{R}^{d} \longrightarrow T_{\pi(\mathfrak{e})}(M)$, and use $\mathfrak{E}_{1}, \ldots, \mathfrak{E}_{d} \in T(\mathcal{O}(M))$ to denote the basic vector fields corresponding to the standard basis $\mathbf{e}_{1}, \ldots, \mathbf{e}_{d}$ in $\mathbb{R}^{d}$. (That is, $\mathfrak{E}_{k}(\mathfrak{e})$ is the unique horizontal element of $T_{\mathfrak{e}}(\mathcal{O}(M))$ such that $d \pi \mathfrak{E}_{k}=\mathfrak{e e}_{k}$. ) (Here, and throughout, we have adopted the notation used in [BC].) Next, let $\lambda_{M}$ be the normalized Riemann measure on $M$, and define the Borel probability measure $\lambda_{\mathcal{O}(M)}$ on $\mathcal{O}(M)$ so that $\lambda_{M}$ is its marginal distribution on $M$ and its conditional distribution is uniform on the fiber $\pi^{-1}(x)$ given that the base point is $x \in M$. In other words, if $x \in U \longmapsto \mathfrak{e}(x) \in \pi^{-1}(x)$ is a smoothly varying, orthonormal frame on the open set $U \subseteq M$ and if $\Psi: \pi^{-1}(U) \longmapsto[0, \infty)$ is Borel measurable, then

$$
\int_{\mathcal{O}(M)} \Psi d \lambda_{\mathcal{O}(M)}=\int_{M}\left(\int_{\mathcal{O}(d)} \Psi\left(R_{\mathcal{O}}(x)\right) d \mathcal{O}\right) \lambda_{M}(d x)
$$

where $R_{\mathcal{O}} \mathfrak{e}$ is the natural right action of $\mathcal{O} \in \mathcal{O}(d)$ on $\mathfrak{e} \in \mathcal{O}(M)$ given by $R_{\mathcal{O}} \mathbf{e v} \equiv \mathfrak{e O} \mathbf{v}, \mathbf{v} \in \mathbb{R}^{d}$, and $d \mathcal{O}$ has been used to denote the normalized

The second author was partially supported by NSF grant 9302709-DMS. 
Haar measure on $\mathcal{O}(d)$. Because $\lambda_{\mathcal{O}(M)}$ is invariant under the flow of each $\mathfrak{E}_{k}$, it is easy to see that, for $\Psi_{1}, \Psi_{2} \in C^{2}(\mathcal{O}(M) ; \mathbb{R})$,

$$
\begin{aligned}
-\int_{\mathcal{O}(M)} \Psi_{1} \Delta \Psi_{2} d \lambda_{\mathcal{O}(M)} & =\sum_{1}^{d} \int_{\mathcal{O}(M)} \mathfrak{E}_{k} \Psi_{1} \mathfrak{E}_{k} \Psi_{2} d \lambda_{\mathcal{O}(M)} \\
& =-\int_{\mathcal{O}(M)} \Psi_{2} \Delta \Psi_{1} d \lambda_{\mathcal{O}(M)}
\end{aligned}
$$

where $\Delta \equiv \sum_{1}^{d} \mathfrak{E}_{k}^{2}$.

Next, let $\left\{\mathcal{P}_{t}: t>0\right\}$ be the Markov semigroup on $C(\mathcal{O}(M) ; \mathbb{R})$ determined by

$$
\mathcal{P}_{t} \Psi-\Psi=\frac{1}{2} \int_{0}^{t} \mathcal{P}_{\tau}(\Delta \Psi) d \tau, \quad t \in(0, \infty) \text { and } \Psi \in C^{2}(\mathcal{O}(M) ; \mathbb{R}) .
$$

As a consequence of (1.1), it is not hard to show that

$$
\int_{\mathcal{O}(M)} \Psi_{1} \mathcal{P}_{t} \Psi_{2} d \lambda_{\mathcal{O}(M)}=\int_{\mathcal{O}(M)} \Psi_{2} \mathcal{P}_{t} \Psi_{1} d \lambda_{\mathcal{O}(M)}
$$

In fact, if $\mathcal{P}_{\mathfrak{e}}$ is the probability measure on $C([0, \infty) ; \mathcal{O}(M))$ determined by

$$
\mathcal{P}_{\mathfrak{e}}(\mathfrak{p}(0)=\mathfrak{e})=1
$$

and

$$
\mathbb{E}^{\mathcal{P}_{\mathfrak{e}}}[\Psi(\mathfrak{p}(T)) \mid \mathfrak{p}(\tau), \tau \in[0, t]]=\left[\mathcal{P}_{T-t} \Psi\right](\mathfrak{p}(t)) \quad\left(\text { a.s., } \mathcal{P}_{\mathfrak{e}}\right)
$$

for $0 \leq t<T$, then $\mathcal{P}_{\mathfrak{e}}$ is a (weakly) continuous function of $\mathfrak{e} \in \mathcal{O}(M)$; and (1.2) plus induction on $n \geq 2$ leads to the conclusion that, for any $0 \leq t_{1}<\cdots \leq t_{n} \leq T<\infty$ and bounded, measurable $\Psi_{1}, \ldots, \Psi_{n}$ on $\mathcal{O}(M)$ :

$$
\begin{aligned}
\mathbb{E}^{\mathcal{P}_{\lambda_{\mathcal{O}}(M)}}\left[\Psi_{1}\left(\mathfrak{p}\left(t_{1}\right)\right)\right. & \left.\cdots \Psi_{n}\left(\mathfrak{p}\left(t_{n}\right)\right)\right] \\
& =\mathbb{E}^{\mathcal{P}_{\lambda_{\mathcal{O}}(M)}}\left[\Psi_{n}\left(\mathfrak{p}\left(T-t_{n}\right)\right) \cdots \Psi_{1}\left(\mathfrak{p}\left(T-t_{1}\right)\right)\right]
\end{aligned}
$$

where

$$
\mathcal{P}_{\lambda_{\mathcal{O}(M)}} \equiv \int_{\mathcal{O}(M)} \mathcal{P}_{\mathfrak{e}} \lambda_{\mathcal{O}(M)}(d \mathfrak{e})
$$


Finally, since, when $\psi \in C^{2}(M ; \mathbb{R}), \Delta \psi \equiv \Delta(\psi \circ \pi)$ is well-defined as a function on $M, P_{t} \psi \equiv \mathcal{P}_{t}(\psi \circ \pi)$ is also well-defined on $M$ for every $\psi \in C(M ; \mathbb{R})$. Hence, $\left\{P_{t}: t>0\right\}$ is a Markov semigroup on $C(M ; \mathbb{R})$. In fact,

$$
P_{t} \psi(x)=\int_{M} \psi(y) p_{t}(x, y) \lambda_{M}(d y)
$$

where $p_{t}(x, y)$ is the fundamental solution (relative to $\lambda_{M}$ ) of the heat equation $\frac{\partial u}{\partial t}=\frac{1}{2} \Delta u$ on $M$.

By standard regularity theory, $p_{t}(x, y)$ is a smooth function of all its variables, by the strong maximum principle, it is strictly positive, and from (1.3), it is clear that $p_{t}(x, y)=p_{t}(y, x)$. After combining these remarks with elementary facts about measures on path spaces, one arrives at the conclusions stated in the following.

1.5 Lemma. Use $\mathfrak{P}(\mathcal{O}(M))$ to denote the space $C([0,1] ; \mathcal{O}(M))$, thought of as a Polish space with the metric of uniform convergence, let $\mathfrak{M}(\mathcal{O}(M))$ be the space of probability measures on $\mathfrak{P}(\mathcal{O}(M))$, thought of as a Polish space with a Lévy metric for the topology of weak convergence, and define $x \in M \longmapsto \mathcal{P}_{x} \in \mathfrak{M}(\mathcal{O}(M))$ so that

$$
\mathcal{P}_{\pi(\mathfrak{e})}=\int_{\mathcal{O}(d)} \mathcal{P}_{R_{\mathcal{O}}} d \mathcal{O}, \quad \mathfrak{e} \in \mathcal{O}(d) .
$$

Then there exists a continuous map

$$
\left(x_{0}, x_{1}\right) \in M^{2} \longmapsto \mathcal{P}_{\left(x_{0}, x_{1}\right)} \in \mathfrak{M}(\mathcal{O}(M))
$$

with the properties

$$
\mathcal{P}_{\left(x_{0}, x_{1}\right)}\left(\pi \circ \mathfrak{p}(0)=x_{0} \text { and } \pi \circ \mathfrak{p}(1)=x_{1}\right)=1 \quad \text { for all }\left(x_{0}, x_{1}\right) \in M^{2},
$$

and, for each $x_{0} \in M$,

$$
\mathcal{P}_{x_{0}}=\int_{\mathfrak{P}(\mathcal{O}(M))} \mathcal{P}_{\left(x_{0}, \pi \circ \mathfrak{p}(1)\right)} \mathcal{P}_{x_{0}}(d \mathfrak{p})=\int_{M} \mathcal{P}_{\left(x_{0}, x\right)} p_{1}\left(x_{0}, x\right) \lambda_{M}(d x) .
$$

In fact, if $\mathcal{B}_{t}$ is the $\sigma$-algebra over $\mathfrak{P}(\mathcal{O}(M))$ generated by $\mathfrak{p} \rightsquigarrow \mathfrak{p}(\tau)$ as $\tau$ runs over $[0, t]$, then, for $t \in[0,1)$ and $A \in \mathcal{B}_{t}$ :

$$
\mathcal{P}_{\left(x_{0}, x_{1}\right)}(A)=\mathbb{E}^{\mathcal{P}_{x_{0}}}\left[\frac{p_{1-t}\left(\pi \circ \mathfrak{p}(t), x_{1}\right)}{p_{1}\left(x_{0}, x_{1}\right)}, A\right] .
$$


Finally, if $\check{\mathfrak{p}}(t)=\mathfrak{p}(1-t)$ for $\mathfrak{p} \in \mathfrak{P}(\mathcal{O}(M))$ and $t \in[0,1]$, then

$$
\mathcal{P}_{\left(x_{0}, x_{1}\right)}(A)=\mathcal{P}_{\left(x_{1}, x_{0}\right)}(\{\mathfrak{p}: \check{\mathfrak{p}} \in A\}), \quad A \in \mathcal{B}_{\mathfrak{P}(\mathcal{O}(M))} .
$$

In words, Lemma 1.5 is saying that $\mathcal{P}_{\left(x_{0}, x_{1}\right)}$ is the distribution of paths $\mathfrak{p} \in \mathfrak{P}(\mathcal{O}(M)))$ under $\mathcal{P}_{\mathfrak{P}}(M)$ given that $\pi \circ \mathfrak{p}(0)=x_{0}$ and $\pi \circ \mathfrak{p}(1)=x_{1}$. Thus, if $P_{\left(x_{0}, x_{1}\right)}$ denotes the marginal distribution of $\mathfrak{p} \rightsquigarrow \pi \circ \mathfrak{p}$ under $\mathcal{P}_{\left(x_{0}, x_{1}\right)}$, then $P_{\left(x_{0}, x_{1}\right)}$ is the distribution of the Brownian motion on $M$ pinned at times 0 and 1 to be at $x_{0}$ and $x_{1}$. In particular, $P_{\left(x_{0}, x_{0}\right)}$ is the distribution of Brownian loops based at $x_{0}$. Finally, (1.9) allows us to interpret $\mathcal{P}_{\left(x_{0}, x_{1}\right)}$ as the distribution of a time-inhomogeneous diffusion. Namely, set

$$
\beta\left(t, \mathfrak{e} ; x_{1}\right)=\nabla_{\mathfrak{e}} \log \left(p_{1-t}\left(\cdot, x_{1}\right)\right), \quad(t, \mathfrak{e}) \in[0,1) \times \mathcal{O}(M),
$$

and

$$
\begin{aligned}
(t, \mathfrak{e}) \in[0,1) \times \mathcal{O}(M) & \longmapsto \\
\quad & \mathfrak{B}\left(t, \mathfrak{e} ; x_{1}\right) \\
& \sum_{1}^{d} \beta\left(t, \mathfrak{e} ; x_{1}\right)_{k} \mathfrak{E}_{k}(\mathfrak{e}) \in T_{\mathfrak{e}}(\mathcal{O}(M)) .
\end{aligned}
$$

Then (1.9) leads to the identification of $\mathcal{P}_{\left(x_{0}, x_{1}\right)}$ as the unique element of $\mathcal{P} \in \mathfrak{P}(\mathcal{O}(M))$ with the properties that $\mathfrak{p}(0)$ under $\mathcal{P}$ is uniformly distributed on $\pi^{-1}\left(x_{0}\right)$ and, for each $\Psi \in C^{2}(\mathcal{O}(M))$,

$$
\left(\Psi(\mathfrak{p}(t))-\int_{0}^{t}\left[\left(\frac{1}{2} \Delta+\mathfrak{B}\left(\tau, \mathfrak{p}(\tau) ; x_{1}\right)\right) \Psi\right](\mathfrak{p}(\tau)) d \tau, \mathcal{B}_{t}, \mathcal{P}\right), \quad t \in[0,1),
$$

is a martingale.

In order to carry out our program, we need to use Itô's representation of the diffusion just described. Thus, let $(\mathbf{H}, \mathfrak{W}, \mu)$ be the standard Wiener of $\mathbb{R}^{d}$-valued paths. That is, $\mathfrak{W}$ is the Banach space of $\mathbf{w} \in C\left([0,1] ; \mathbb{R}^{d}\right)$ with $\mathbf{w}(0)=\mathbf{0}$ and $\|\mathbf{w}\|_{\mathfrak{W}}=\sup _{t \in[0,1]}|\mathbf{w}(t)|, \mathbf{H}$ is the Hilbert space of absolutely continuous $\mathbf{h} \in \mathfrak{W}$ with $\|\mathbf{h}\|_{\mathbf{H}}=\|\dot{\mathbf{h}}\|_{L^{2}\left([0,1] ; \mathbb{R}^{d}\right)}<\infty$, and $\mu$ is the Borel probability on $\mathfrak{W}$ whose Fourier transform $\hat{\mu}: \mathfrak{W}^{*} \longrightarrow \mathbb{C}$ is given by

$$
\begin{aligned}
\hat{\mu}(\alpha) & \equiv \mathbb{E}^{\mu}\left[e^{\sqrt{-1}\langle\mathbf{w}, \alpha\rangle}\right] \\
& =\exp \left[-\frac{\left\|\mathbf{h}_{\alpha}\right\|_{\mathbf{H}}^{2}}{2}\right]=\exp \left[\iint_{[0,1]^{2}} s \wedge t(\alpha(d s), \alpha(d t))_{\mathbb{R}^{d}}\right],
\end{aligned}
$$


where we have identified $\mathfrak{W}^{*}$ with the space of totally finite, $\mathbb{R}^{d}$-valued measures $\alpha$ on $[0,1]$ and we have embedded $\mathfrak{W}^{*}$ in $\mathbf{H}$ via the map $\alpha \rightsquigarrow \mathbf{h}_{\alpha}$ where $\dot{\mathbf{h}}_{\alpha}(t)=\alpha((t, 1])$. Next, set $\tilde{\mathfrak{W}}=\mathcal{O}(d) \times \mathfrak{W}$, and use $\tilde{\mu}$ on $\tilde{\mathfrak{W}}$ to denote the product of Haar measure with $\mu$. Finally, for a given $\left(x_{0}, x_{1}\right) \in M^{2}$, choose $\mathfrak{e}_{0} \in \pi^{-1}\left(x_{0}\right)$, and define $\mathfrak{F}_{\left(\mathfrak{e}_{0}, x_{1}\right)}:[0,1) \times \tilde{\mathfrak{W}} \longrightarrow \mathcal{O}(M)$ to be the $\tilde{\mu}^{-}$ almost surely unique progressively measurable solution to the Stratonovich stochastic differential equation

$$
\begin{aligned}
d \mathfrak{F}_{\left(\mathfrak{e}_{0}, x_{1}\right)}(t, \tilde{\mathbf{w}})=\sum_{1}^{d} \mathfrak{E}_{k} & \left(\mathfrak{F}_{\left(\mathfrak{e}_{0}, x_{1}\right)}(t, \tilde{\mathbf{w}})\right) \circ d \mathbf{w}(t)_{k} \\
& +\mathfrak{B}\left(t, \mathfrak{F}_{\left(\mathfrak{e}_{0}, x_{1}\right)}(t, \tilde{\mathbf{w}}) ; x_{1}\right) d t, \quad t \in[0,1),
\end{aligned}
$$

with $\mathfrak{F}_{\left(\mathfrak{e}_{0}, x_{1}\right)}(0, \tilde{\mathbf{w}})=R_{\mathcal{O}} \mathfrak{e}_{0}$ for $\tilde{\mu}$-almost every $\tilde{\mathbf{w}}=(\mathcal{O}, \mathbf{w})$.

As an application of Itô's formula for Stratonovich integrals and the preceding martingale characterization of $\mathcal{P}_{\left(x_{0}, x_{1}\right)}$, one can make the following identification.

1.14 Theorem. Given $\left(x_{0}, x_{1}\right) \in M^{2}$ and $\mathfrak{e}_{0} \in \pi^{-1}\left(x_{0}\right)$, the solution $\mathfrak{F}_{\left(\mathfrak{e}_{0}, x_{1}\right)}(\cdot, \tilde{\mathbf{w}})$ to $(1.13)$ has a limit

$$
\mathfrak{F}_{\left(\mathfrak{e}_{0}, x_{1}\right)}(1, \tilde{\mathbf{w}}) \equiv \lim _{t \nearrow 1} \mathfrak{F}_{\left(\mathfrak{e}_{0}, x_{1}\right)}(t, \tilde{\mathbf{w}}) \quad \text { for } \tilde{\mu} \text {-almost every } \tilde{\mathbf{w}} \in \tilde{\mathfrak{W}}
$$

Moreover, $\mathcal{P}_{\left(x_{0}, x_{1}\right)}$ is the distribution of $\tilde{\mathbf{w}} \in \tilde{\mathfrak{W}} \longmapsto \mathfrak{F}_{\left(\mathfrak{e}_{0}, x_{1}\right)}(\cdot, \tilde{\mathbf{w}}) \in$ $\mathfrak{P}(\mathcal{O}(M))$ under $\tilde{\mu}$. In particular, $\pi\left(\mathfrak{F}_{\left(\mathfrak{e}_{0}, x_{1}\right)}(1, \tilde{\mathbf{w}})\right)=x_{1}$ (a.s., $\left.\tilde{\mu}\right)$.

\section{An integration by parts formula}

In order to formulate our main result, we have to introduce some additional notation. In the first place, we use $\omega$ and $\phi$ to denote, respectively, the $\mathbb{R}^{d}$ valued solder form and the $\mathfrak{o}(d)$-valued connection 1-form on $\mathcal{O}(M)$. Next, choose and fix a pair of points $x_{0}$ and $x_{1}$ in $M$, set

$$
\mathfrak{P}_{\left(x_{0}, x_{1}\right)}(\mathcal{O}(M))=\left\{\mathfrak{p} \in \mathfrak{P}(\mathcal{O}(M)): \pi \circ \mathfrak{p}(0)=x_{0} \text { and } \pi \circ \mathfrak{p}(1)=x_{1}\right\} \text {, }
$$

and define $C^{1}(\mathbb{R} ; \mathfrak{P}(\mathcal{O}(M)))$ to be the space of continuously differentiable maps $s \in \mathbb{R} \longmapsto \mathfrak{P}(s) \in \mathfrak{P}_{\left(x_{0}, x_{1}\right)}(\mathcal{O}(M))$ with properties that

$$
t \in[0,1] \longmapsto\left[\omega\left(\mathfrak{P}^{\prime}(0)\right)\right](t) \equiv \omega\left(\left[\mathfrak{P}^{\prime}(0)\right](t)\right) \in \mathbb{R}^{d}
$$

is an element of $\mathbf{H}_{0} \equiv\{\mathbf{h} \in \mathbf{H}: \mathbf{h}(1)=\mathbf{0}\}$ 
and

$$
t \in[0,1] \longmapsto\left[\phi\left(\mathfrak{P}^{\prime}(0)\right)\right](t) \equiv \phi\left(\left[\mathfrak{P}^{\prime}(0)\right](t)\right) \in \mathfrak{o}(d)
$$

is continuous and vanishes at $t=0$.

Second, given a separable Hilbert space $X$, let $\mathcal{M}_{\mathrm{c}}(X)$ be the space of totally finite, $X$-valued Borel measures on $(0,1)$ with compact support, and give $\mathcal{M}_{\mathrm{c}}(X)$ the strong topology. If $F \in C\left(\mathfrak{P}_{\left(x_{0}, x_{1}\right)}(\mathcal{O}(M)) ; X\right)$ and there exists an $\mathfrak{D} F \in C(\mathfrak{P}(\mathcal{O}(M)) ; \mathbf{H} \otimes X)$ and a $\Lambda F \in C\left(\mathfrak{P}(\mathcal{O}(M)) ; \mathcal{M}_{\mathrm{c}}(\mathfrak{o}(d) \otimes\right.$ $X)$ ) with the property that

$$
\left.\frac{d}{d s} F(\mathfrak{P}(s))\right|_{s=0}=\left(\omega\left(\mathfrak{P}^{\prime}(0)\right), \mathfrak{D} F(\mathfrak{P}(0))\right)_{\mathbf{H}}+\left\langle\phi\left(\mathfrak{P}^{\prime}(0)\right), \Lambda F(\mathfrak{P}(0))\right\rangle,
$$

we will write $F \in C^{1}\left(\mathfrak{P}_{\left(x_{0}, x_{1}\right)}(\mathcal{O}(M)) ; X\right)$, where we have used $\langle\cdot, \cdot\rangle$ to denote the duality relation induced by integrating functions with respect to measures. It is a simple matter to check that both $\mathfrak{D} F$ and $\Lambda F$ are uniquely determined.

Actually, our real interest is in functions defined not on $\mathfrak{P}_{\left(x_{0}, x_{1}\right)}(\mathcal{O}(M))$ but on

$$
\mathfrak{P}_{\left(x_{0}, x_{1}\right)}(M) \equiv\left\{p \in C([0,1] ; M): p(0)=x_{0} \text { and } p(1)=x_{1}\right\} .
$$

Thus, define $C^{1}\left(\mathfrak{P}_{\left(x_{0}, x_{1}\right)}(M) ; X\right)$ to be the space of $f \in C\left(\mathfrak{P}_{\left(x_{0}, x_{1}\right)}(M) ; X\right)$ with the property that $f \circ \pi \in C^{1}\left(\mathfrak{P}_{\left(x_{0}, x_{1}\right)}(\mathcal{O}(M)) ; X\right)$. Clearly, $\Lambda(f \circ \pi) \equiv$ $\mathbf{0}$, and therefore

$$
\left.\frac{d}{d s} f \circ \pi(\mathfrak{P}(s))\right|_{s=0}=\left(D f((\mathfrak{P}(0))), \omega\left(\mathfrak{P}^{\prime}(0)\right)\right)_{\mathbf{H}} \quad \text { where } D f \equiv \mathfrak{D}(f \circ \pi) .
$$

Next, let $\mathbf{D}^{1}\left(\mathfrak{P}_{\left(x_{0}, x_{1}\right)}(M) ; X\right)$ denote the space of $f \in C^{1}\left(\mathfrak{P}_{\left(x_{0}, x_{1}\right)}(M) ; X\right)$ for which there exists a continuous map $\lambda_{f}: \mathfrak{P}(\mathcal{O}(M)) \longrightarrow \mathcal{M}_{\mathrm{c}}\left(\mathbb{R}^{d} \otimes X\right)$ such that

$$
[D f(\mathfrak{p})](t)=\int_{[0, \infty)} t \wedge \tau \lambda_{f}(\mathfrak{p})(d \tau), \quad \mathfrak{p} \in \mathfrak{P}_{\left(x_{0}, x_{1}\right)}(\mathcal{O}(M)) \text { and } t \in[0,1] .
$$

It is easy to check that:

$$
\pi \circ \mathfrak{q}=\pi \circ \mathfrak{p} \Longrightarrow \lambda_{f}(\mathfrak{q})(d \tau)=\mathfrak{q}(\tau)^{-1} \mathfrak{p}(\tau) \lambda_{f}(\mathfrak{p})(d \tau)
$$

Our basic integration by parts formula is contained in the following. 
2.2 Theorem. Let Ric : $\mathcal{O}(M) \longrightarrow \mathbb{R}^{d} \otimes \mathbb{R}^{d}$ denote the Ricci curvature, set

$$
\mathcal{H}\left(t, \mathfrak{e} ; x_{1}\right)=\operatorname{Hess}\left(\log p_{1-t}\left(\cdot, x_{1}\right)\right)(\mathfrak{e}) \quad \text { for }(t, \mathfrak{e}) \in[0,1) \times \mathcal{O}(M)
$$

and define

$$
\begin{aligned}
& {\left[\mathfrak{R}\left(\mathfrak{e}, x_{1}\right) \mathbf{h}\right](t)=\dot{\mathbf{h}}(t)+\widetilde{\operatorname{Ric}}\left(t, \mathfrak{e}, x_{1}\right) \mathbf{h}(t)} \\
& \text { where } \widetilde{\operatorname{Ric}}\left(t, \mathfrak{e}, x_{1}\right) \equiv \operatorname{Ric}(\mathfrak{e})-\mathcal{H}\left(t, \mathfrak{e} ; x_{1}\right), \quad \mathbf{h} \in \mathbf{H} .
\end{aligned}
$$

Then there exists a $C<\infty$ for which

$$
\mathbb{E}^{\tilde{\mu}}\left[\int_{0}^{1}\left|\mathcal{H}\left(\tau, \mathfrak{F}_{\left(x_{0}, x_{1}\right)}(\tau) ; x_{1}\right) \mathbf{h}(\tau)\right|^{2} d \tau\right] \leq C\|\mathbf{h}\|_{\mathbf{H}}^{2}, \quad \mathbf{h} \in \mathbf{H}_{0}
$$

Moreover, for $f, g \in D^{1}\left(\mathfrak{P}_{\left(x_{0}, x_{1}\right)}(M) ; \mathbb{R}\right)$ and $\mathbf{h} \in \mathbf{H}_{0}$ :

$$
\begin{aligned}
& \int_{\mathcal{O}(M)}(D f(\mathfrak{p}), \mathbf{h})_{\mathbf{H}} g \circ \pi(\mathfrak{p}) \mathcal{P}_{\left(x_{0}, x_{1}\right)}(d \mathfrak{p}) \\
&=-\int_{\mathcal{O}(M)} f \circ \pi(\mathfrak{p})(D g(\mathfrak{p}), \mathbf{h})_{\mathbf{H}} \mathcal{P}_{\left(x_{0}, x_{1}\right)}(d \mathfrak{p}) \\
&+\mathbb{E}^{\tilde{\mu}}\left[\int_{0}^{1}\left(\left[\mathfrak{R}\left(\mathfrak{F}_{\left(x_{0}, x_{1}\right)}(\tau), x_{1}\right) \mathbf{h}\right](\tau), d \mathbf{w}(\tau)\right)_{\mathbb{R}^{d}}(f g) \circ \mathfrak{F}_{\left(x_{0}, x_{1}\right)}\right] .
\end{aligned}
$$

Our proof of Theorem 2.2 is based on the same general strategy as our proof of Theorem 4.9 in [ES]. Namely, we introduce a perturbation procedure of the same sort as the one described in Theorems 2.5 and 3.1 of [ES]. This procedure yields, for $\tilde{\mu}$-almost every $\tilde{\mathbf{w}} \in \tilde{\mathfrak{W}}$, a differentiable map $s \in \mathbb{R} \longmapsto\left[\mathfrak{F}_{\left(x_{0}, x_{1}\right)}(\cdot, \tilde{\mathbf{w}})\right](s) \in \mathfrak{P}(\mathcal{O}(M))$ with the properties that:

(1) $\left[\mathfrak{F}_{\left(x_{0}, x_{1}\right)}(\cdot, \tilde{\mathbf{w}})\right](0)=\mathfrak{F}_{\left(x_{0}, x_{1}\right)}(\cdot, \tilde{\mathbf{w}})$,

(2) $\omega\left(\frac{d}{d s}\left[\mathfrak{F}_{\left(x_{0}, x_{1}\right)}(t, \tilde{\mathbf{w}})\right](s)\right)=\mathbf{h}(t)$,

(3) for each $s$, the distribution of $\tilde{\mathbf{w}} \in \tilde{\mathfrak{W}} \longmapsto\left[\mathfrak{F}_{\left(x_{0}, x_{1}\right)}(\cdot, \tilde{\mathbf{w}})\right](s) \in$ $\mathfrak{P}(\mathcal{O}(M))$ under $\tilde{\mu}$ is equivalent to that of $\tilde{\mathbf{w}} \rightsquigarrow \mathfrak{F}_{\left(x_{0}, x_{1}\right)}(\cdot, \tilde{\mathbf{w}})$, and the Radon-Nikodym derivative is a smooth function of $s$.

The most interesting new challenge comes from the need to handle the singularity at $t=1$ of the vector field $\mathfrak{B}\left(t, \mathfrak{e} ; x_{1}\right)$, and this is precisely the reason why one wants an estimate like the one in (2.3). Our derivation of (2.3) is based on two observations. In the first place, by taking advantage of 
the reversibility contained in (1.10), one can show that, for each $p \in[1, \infty)$, there is a $C<\infty$ such that

$$
\mathbb{E}^{\tilde{\mu}}\left[\operatorname{dist}\left(\mathfrak{F}_{\left(x_{0}, x_{1}\right)}(t), x_{1}\right)^{2 p}\right] \leq C_{p}(1-t)^{p}, \quad t \in[0,1) .
$$

Secondly, one must check that there is a $C<\infty$ for which one has the estimate

$$
\left\|\mathcal{H}\left(t, \mathfrak{e} ; x_{1}\right)\right\|_{\text {H.S. }} \leq C\left(\frac{1}{1-t}+\frac{\operatorname{dist}\left(\pi(\mathfrak{e}), x_{1}\right)^{2}}{(1-t)^{2}}\right) .
$$

The integration by parts formula in (2.4) can be used to introduce a Laplacian on the space of pinned loops. For this purpose, take

$$
\mathbf{D}^{2}\left(\mathfrak{P}_{\left(x_{0}, x_{1}\right)}(M) ; X\right)
$$

to stand for the space of $f \in \mathbf{D}^{1}\left(\mathfrak{P}_{\left(x_{0}, x_{1}\right)}(M) ; X\right)$ for which

$$
D f \in C^{1}\left(\mathfrak{P}_{\left(x_{0}, x_{1}\right)}(\mathcal{O}(M)) ; \mathbf{H} \otimes X\right),
$$

and observe that

$$
\langle\phi, \Lambda D f(\mathfrak{p})\rangle(t)=-\int_{[0, \infty)} t \wedge \tau \phi(\tau) \lambda_{f}(\mathfrak{p})(d \tau) .
$$

Finally, use $\mathbf{D}_{\mathrm{b}}^{2}(\mathfrak{P}(\mathcal{O}(M)) ; X)$ to denote the space of

$$
f \in \mathbf{D}^{2}\left(\mathfrak{P}_{\left(x_{0}, x_{1}\right)}(\mathcal{O}(M)) ; X\right)
$$

for which the total variation of $\lambda_{f}$ is uniformly bounded and, for any orthonormal basis $\left\{\mathbf{h}_{\alpha}\right\}$ in $\mathbf{H}_{0}$,

$$
\sum_{\alpha} \sup _{\mathfrak{p} \in \mathfrak{P}_{\left(x_{0}, x_{1}\right)}(\mathcal{O}(M))}\left\|\left(\mathbf{h}_{\alpha} \otimes \mathbf{h}_{\alpha}, \mathfrak{D} D f(\mathfrak{p})\right)_{\mathbf{H} \otimes \mathbf{H}}\right\|_{X}<\infty .
$$

Clearly, when $f \in D_{\mathrm{b}}^{2}\left(\mathfrak{P}_{\left(x_{0}, x_{1}\right)}(M) ; X\right)$,

$$
\mathfrak{p} \in \mathfrak{P}(\mathcal{O}(M)) \longmapsto \operatorname{Trace}_{\mathbf{H}}(\mathfrak{D} D f(\mathfrak{p})) \equiv \sum_{\alpha}\left(\mathbf{h}_{\alpha} \otimes \mathbf{h}_{\alpha}, \mathfrak{D} D f(\mathfrak{p})\right)_{\mathbf{H} \otimes \mathbf{H}} \in X
$$

is a bounded continuous function which is independent on the choice of orthonormal basis $\left\{\mathbf{h}_{\alpha}\right\}$. Moreover,

$$
\begin{aligned}
& \pi \circ \mathfrak{p}=\pi \circ \mathfrak{q} \text { and } \mathfrak{q}(t)^{-1} \mathfrak{p}(t) \text { independent of } \mathrm{t} \\
& \qquad \Longrightarrow \operatorname{Trace}_{\mathbf{H}}(\mathfrak{D} D f(\mathfrak{q}))=\operatorname{Trace}_{\mathbf{H}}(\mathfrak{D} D f(\mathfrak{p})) .
\end{aligned}
$$

Starting from (2.4), one can easily (cf. the proof of Theorem 4.30 in [ES]) derive the following. 
2.5 Theorem. Define $\tilde{\mathbf{w}} \in \tilde{\mathfrak{W}} \longmapsto \mathfrak{U}_{\left(x_{0}, x_{1}\right)}(\tilde{\mathbf{w}}) \in C([0,1] ; \mathbb{R})$ by

$$
\begin{aligned}
& {\left[\mathfrak{U}_{\left(x_{0}, x_{1}\right)}(\tilde{\mathbf{w}})\right](t)=\mathbf{w}(t)-\int_{0}^{t} \tau \operatorname{Ric}\left(\mathfrak{F}_{\left(x_{0}, x_{1}\right)}(\tau, \tilde{\mathbf{w}})\right) d \mathbf{w}(\tau)} \\
& -\frac{1}{4} \int_{0}^{t} \tau \nabla_{\mathfrak{F}_{\left(x_{0}, x_{1}\right)}(\tau, \tilde{\mathbf{w}})} \kappa d \tau+\frac{1}{2} \int_{0}^{1} t \wedge \tau \widetilde{\operatorname{Ric}}\left(t, \mathfrak{F}_{\left(x_{0}, x_{1}\right)}(\tau, \tilde{\mathbf{w}}), x_{1}\right) d \mathbf{w}(\tau),
\end{aligned}
$$

where $\kappa$ is the scalor curvature; and, for $f \in \mathbf{D}^{2}\left(\mathfrak{P}_{\left(x_{0}, x_{1}\right)}(M) ; \mathbb{R}\right)$, set

$$
\begin{aligned}
{\left[\mathfrak{L}_{\left(x_{0}, x_{1}\right)} f\right](\tilde{\mathbf{w}})=\operatorname{Trace}_{\mathbf{H}_{0}} } & \left(\mathfrak{D} D f\left(\mathfrak{F}_{\left(x_{0}, x_{1}\right)}(\cdot, \tilde{\mathbf{w}})\right)\right) \\
- & \int_{[0,1]}\left[\mathfrak{U}_{\left(x_{0}, x_{1}\right)}(\tilde{\mathbf{w}})\right](t)\left[\lambda_{f}\left(\mathfrak{F}_{\left(x_{0}, x_{1}\right)}(\cdot, \tilde{\mathbf{w}})\right)\right](d t)
\end{aligned}
$$

for $\tilde{\mathbf{w}} \in \tilde{\mathfrak{W}}$. Then, for all $f, g \in \mathbf{D}_{\mathrm{b}}^{2}\left(\mathfrak{P}_{\left(x_{0}, x_{1}\right)}(M) ; \mathbb{R}\right)$,

$$
\begin{aligned}
-\int_{\mathfrak{P}_{\left(x_{0}, x_{1}\right)}(\mathcal{O}(M))} & (D f(\mathfrak{p}), D g(\mathfrak{p}))_{\mathbf{H}_{0}} \mathcal{P}_{\left(x_{0}, x_{1}\right)}(d \mathfrak{p}) \\
= & \int_{\tilde{\mathfrak{W}}} g \circ \pi\left(\mathfrak{F}_{\left(x_{0}, x_{1}\right)}(\cdot, \tilde{\mathbf{w}})\right)\left[\mathfrak{L}_{\left(x_{0}, x_{1}\right)} f\right](\tilde{\mathbf{w}}) \tilde{\mu}(d \tilde{\mathbf{w}}) .
\end{aligned}
$$

In particular, $\mathfrak{L}_{\left(x_{0}, x_{1}\right)}$ is a nonpositive, symmetric operator for which

$$
\int_{\mathfrak{P}_{\left(x_{0}, x_{1}\right)}(\mathcal{O}(M))}(D f(\mathfrak{p}), D g(\mathfrak{p}))_{\mathbf{H}_{0}} \mathcal{P}_{\left(x_{0}, x_{1}\right)}(d \mathfrak{p})
$$

is the associated Dirichlet form.

\section{References}

[BC] R. Bishop and R. Crittenden, Geometry of Manifolds, Academic Press, N.Y., 1964.

[D1] B. Driver, A Cameron-Martin type quasi-invariance theorem for Brownian motion on compact Riemannian manifolds, Jour. Funct. Anal. 110 (1992), 272-376.

[D2] _ A Cameron-Martin type quasi-invariance theorem for pinned Brownian motion on compact Riemannian manifolds, Trans. Amer. Math. Soc. 342 (1994), 375-395.

[ES] O. Enchev and D. Stroock, Towards a Riemannian geometry on the path space over a Riemannian manifold (to appear in Jour. Funct. Anal.).

Department of Mathematics, Boston University, Boston, M A 02215

E-mail address: ogi@math.bu.edu

M IT, 2-272, CAMBRIDGe, M A 02139

E-mail address:dws@math.mit.edu 